\title{
Recovery of Myocardial Kinematic Function without the Time History of External Loads
}

\author{
Heye Zhang, Bo Li, Alistair A. Young, and Peter J. Hunter \\ Bioengineering Institute, University of Auckland, Auckland 1142, New Zealand \\ Correspondence should be addressed to Heye Zhang, heye.zhang@auckland.ac.nz
}

Received 30 April 2009; Accepted 24 June 2009

Academic Editor: João Manuel R. S. Tavares

Copyright () 2010 Heye Zhang et al. This is an open access article distributed under the Creative Commons Attribution License, which permits unrestricted use, distribution, and reproduction in any medium, provided the original work is properly cited.

\begin{abstract}
A time-domain filtering algorithm is proposed to recover myocardial kinematic function using output-only measurements without the time history of external loads. The main contribution of this work is that the overall effect of all the external loads on the myocardium is treated as a random variable disturbed by the Gaussian white noise because the external loads of the myocardium are usually unknown in practical exercises. The kernel of our proposed algorithm is an iterative, multiframe, and sequential filtering procedure consisting of a Kalman filter and a least-squares filter. In our proposed implementation, the initial guess of myocardial kinematic function and residual innovation of all the state variables are first computed using a Kalman filter via state space equations only driven by the Gaussian white noise, and then the residual innovation is fed into a least-squares filter to estimate the total external loads of the myocardium. In the end, the initial guess of myocardial kinematic function is corrected using external loads provided by the least-squares filter. After the introduction of the whole structure of our algorithm, we demonstrate the ability of the framework on synthetic data and MR image sequences.
\end{abstract}

\section{Introduction}

Ischemic heart disease (IHD), or myocardial ischaemia, is a heart disease characterized by restricted blood supply to a certain area of muscle wall of the heart (myocardium), usually due to the blockage or shrinkage of the coronary artery. The restricted blood supply in the particular area of the myocardium can cause dysfunction or even permanent damage (infarction) if left untreated. In daily clinical practice, because of recent technological advances in cardiac imaging modalities, particularly magnetic resonance imaging (MRI), multislice computed tomography (CT), and echocardiography, assessment of the regional kinematic function of the myocardium has been largely applied to estimate the location of infarction and evaluate the seriousness of infarction by clinical specialists. In the medical image community, the idea to indicate IHD or infarction accurately via quantification of myocardial kinematic function has stimulated a huge number of computing algorithms to overcome practical difficulties, for example, relatively sparse spatial resolution and low temporal sampling rate of current imaging modalities. Most early works utilize pixel intensities to evaluate myocardial kinematic function using one or even more imaging modalities [1-3], but the performance of these approaches varies largely because of the quality of the image. Recently, more and more feature points, such as tagging lines [4-6] or boundaries $[7,8]$, have been introduced as extra constraints to enhance the performance of intensitybased approaches, however, these intensity-based approaches still suffer from noise in the image data. The recovery of a dense motion field and deformation parameters for the entire myocardium from a sparse set of image-derived displacements/veolcities seems an ill-posed problem which needs more physically meaningful constraints to obtain a unique solution in some optimal sense.

Therefore, a large number of strategies have been developed over the past two decades to introduce a variety of physically meaningful constraints into myocardial motion analysis, including notable examples of mathematically motivated regularization [9], deformable superquadrics [10], spatiotemporal B-Spline [11], Fisher estimator with smoothness and incompressibility assumptions [12], as well as finite-element method- (FEM-) based modal analysis [1315]. From the introduction of biomechanical model-based 
constraint [16-18] into the medical image community, the biomechanical model has attracted great attention because of its physiologically meaningful representation of myocardial dynamics. Contrary to previous frame-to-frame strategies with biomechanical model-based constraint, multiframe analysis strategies are gradually accepted since the periodic nature of myocardial dynamics is widely recognized. A number of image analysis works are motivated to adopt different biomechanical models from system point of view $[19,20]$. In [21], the authors adopt system control theory [22] into medical image analysis by establishing a biomechanical model-based state-space framework for the multiframe estimation of the periodic myocardial motion: "the physical constraints take the role of the spatial regulator of the myocardial behavior and spatial filter/interpolator of the data measurements, while techniques from statistical filtering theory impose spatiotemporal constraints to facilitate the incorporation of multiframe information to generate optimal estimates of the myocardial kinematics in 2D." The authors of [21] also apply a similar state-space filtering structure to estimate parameters of biomechanical models and myocardial motion simultaneously in [23, 24], but the filtering techniques in $[23,24]$ are realized by the extended Kalman filter and $H_{\infty}$ filter, respectively. However, the computation of the Kalman filter has prohibited its popularity in 3D motion analysis. Thus, a reduced-rank Kalman filter was proposed to reduce the computation and estimate 3D myocardial kinematic function using a small number of principal modal components in [25]. In spite of the potential advantage of computational speed in [25], the effect of infarction might not be reflected by a small number of principal modal components because the influence of infarction to the whole myocardium could be localized and small. In the most recent works feedback, control techniques are also applied to estimate cardiac motion with a collocate state estimator [26] and parameters of biomechanical model using an extended Kalman filter [27] or $H_{\infty}$ filter [28] seperately. Despite sharing the same origin from the control theory, techniques in [26-28] still belong to the class of "deterministic models" as defined in [29], which are different from "stochastic models" in [21, 24]. However, the importance of external loads to the biomechanical model has not been addressed in multiframe medical image analysis in spite of a simple fact that the loading condition of each patient is not the same. Though different biomechanical constraints, from isotropic material to anisotropic material or from small deformation to large deformation, have been applied to myocardial motion analysis, most of works assume external loads of the biomechanical model as implicitly available forces from the image-derived boundary [18, 23, 24] or from a priori knowledge [26-28, 30]. In [31], external loads are obtained by a weighting between boundary-derived force from images and the electrical force from simulation. All these deterministic treatments of external loads are not patient-specific and a minor error in external loads might alert the dynamics of the same biomechanical model largely, which would damage the positive effect of model constraint eventually. In [32], a frame-to-frame statistical EM algorithm is applied to estimate the active forces, strains, and stresses together despite the fact that the active forces are timevarying after the displacements of the myocardium are reconstructed by using the MRI-SPAMM tagging technique and a deformable model from images.

Inspired by the work [33] of input estimation in the inverse heating problem without the time history of external input, we proposed a biomechanical constrained sequential filtering framework which performs multiframe estimation of the nonrigid myocardial kinematic function and external loads of system simultaneously from medical image sequence. Our work is also developed from earlier works like the state-space-based motion recovery algorithms with the external loads constructed from boundaries [21, 24] and model-based filtering framework with external loads simulated from an electromechanical coupling model [30]. Contrary to previous approaches using deterministic approximation of external loads, our proposed framework treats external loads as a stochastic input after the biomechanical model is converted into a stochastic state-space representation, which is more rational because of largely unknown knowledge of external loads of each patient's heart. Since the external loads in our approach are treated as a stochastic variable, we start to call external loads as input forces from here, which is a proper description in the stochastic control theory [22]. Therefore, the main difference of our work to previous multiframe estimation efforts is that rather than making ad hoc mathematical assumptions on the behavior of input forces, we allow uncertainties inside input forces because of unobservable loading condition of the heart, which is a better description of clinical situation from the stochastic control theory point of view. To achieve optimal estimation, we cyclically feed the updated estimation of input forces and imaging-derived data into the filtering framework until reaching largely data-driven convergence: a Kalman filter is first used to generate the residual innovation sequences without input forces, followed by a recursive least-square filter which is derived to use the sequences of residual innovation to compute values of input forces to the myocardium. Finally, myocardial kinematic function can be recovered by using the estimated input forces.

The outline of the paper is as follows. Section 2 describes the underlying myocardial dynamics, that is, the state-space representation of biomechanical model. The combination of the Kalman filter and the recursive least-square filter to recover input forces and correct the estimation of myocardial kinematic function is introduced in Section 3. We finally evaluate our algorithm in Section 4 and present the corresponding discussion and conclusion in Section 5.

\section{Representation of Myocardial Dynamics}

As a rule of thumb, the heart is a complex mechanical system in terms of large deformation and complicated material properties [34]. Many sophisticated models have been built over tremendous experiments to reproduce the behavior of the heart [34]. However, the complexities of these models limit their performance in understanding patient's data because of high computational requirements. Furthermore, 


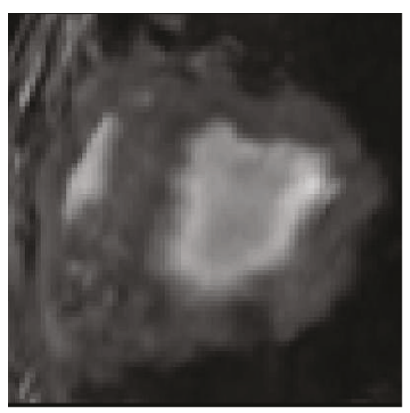

(a)

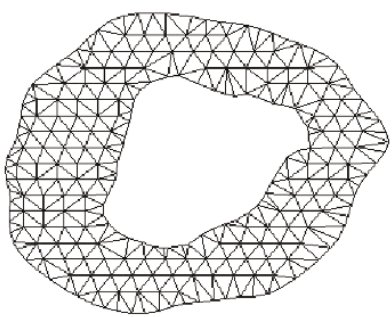

(b)

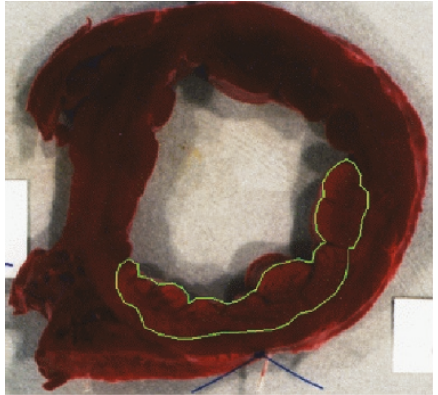

(c)

FIgure 1: (a) Mid-ventricle MRI slice of a canine heart, (b) FEM representation of left ventricle constructed from MRI slice, and (c) TTC stained postmortem myocardium with the infarcted tissue highlighted.

some errors of initialization can be accumulated and amplified through the model dynamics because of the deterministic nature of these models. The purpose of this paper is to build a stochastic representation of myocardial dynamics, which relaxes the requirement of complexity and accuracy of the model by introducing uncertainties into the biomechanical model. In the following subsections, a deterministic the finite-element representation of the myocardium using the law of linear elasticity is built, and then this representation plus its relation of measurement will be converted into stochastic state space equations. The reason to choose linear elasticity in this work is to construct a rationally realistic and computationally feasible analysis framework using imaging data and other available measurements, the structure, dynamics, and material of the myocardium. In the stochastic representation, the model errors, which could be caused by an imperfect model, insufficient discretization, or incorrect initialization, and measurement errors are properly addressed as noise terms in each state space equation. It is noted that other computational cardiac mechanics of materials also can be adopted into this stochastic framework.

2.1. Law of Linear Elasticity. In the current 2D implementation, we adopt an isotropic linear elastic material property, where the stress and strain relationship obeys Hooke's law [35], to approximate myocardial dynamics:

$$
\sigma=S \varepsilon
$$

where $\sigma$ is the stress tensor and $\varepsilon$ is the strain tensor.

In the law of mechanical deformation, the infinitesimal strain tensor or Cauchy's strain tensor will be used to describe the deformation of an object with elastic material properties [35]. The infinitesimal strain tensor is calculated by

$$
\varepsilon=\left[\begin{array}{c}
\frac{\partial u_{x}}{\partial x} \\
\frac{\partial u_{y}}{\partial y} \\
\frac{\partial u_{x}}{\partial y}+\frac{\partial u_{y}}{\partial y}
\end{array}\right]
$$

where $u_{x}$ is the displacement along $x$ axis and $u_{y}$ is the displacement along $y$ axis in location $(x, y)$. In our 2D implementation, the plane strain condition is assumed. So the material constitutive matrix $S$ is

$$
S=\frac{E}{(1+\nu)(1-2 v)}\left[\begin{array}{ccc}
1-\nu & \nu & 0 \\
\nu & 1-\nu & 0 \\
0 & 0 & \frac{1-2 \nu}{2}
\end{array}\right],
$$

where $E$ is Young's modulus and $v$ is Poisson's ratio [35]. In previous work [36], the values of these two myocardial material variables are specified as $E=75 \mathrm{kpa}$ and $v=0.47$. In the following subsection, the finite-element representation, numerical discretization of the myocardium will be built using the material constitutive law established in the following subsection.

2.2. Finite-Element Mesh of Myocardium. The finite-element method has been a standard numerical method in solving partial differential equations. In this implementation, a triangular mesh is generated to represent a $2 \mathrm{D}$ myocardial slice, which is segmented in the MR images by a spatialtemporal active region model strategy [37]. The displacements of all the vertices in the mesh are calculated by an automatic nonrigid registration algorithm [38]. The finiteelement mesh over one image plane and corresponding MR image are illustrated by Figure 1. Over the constraint of linear isotropic linear elasticity and the linear triangular finiteelement mesh, the nodal displacement-based governing dynamic equation of each element is established under the principle of minimum potential energy. These equations finally can be assembled together in matrix form as [35]

$$
M \ddot{U}(t)+C \dot{U}(t)+K U(t)=R(t),
$$

where $M, C$, and $K$ are the mass, damping, and stiffness matrices, respectively, $R$ is the load vector, and $U$ is the displacement vector. Also $M$ is a known function of material density and is assumed temporally constant for incompressible material, $K$ is a function of material constitutive law, and is related to Young's modulus and Poisson's ratio which are again assumed constant. Finally, $C$ is frequency dependent, 
and we assume Rayleigh damping $C=\alpha M+\beta K$ with small constant $\alpha$ and $\beta$ for the low damping myocardial tissue [35].

We need to point out that the input forces $R$, which are driven by electrical excitations and blood pressure, of the cardiac system are highly complicated. In clinical practice, observations of intraventricular blood pressures are too sparse and noisy. In spite of many efforts which are aimed to recover intracardiac electrical excitations from body surface potentials [39], the coupling of electrical excitations and active forces remain unknown in clinical practice because of difficulties. So it is a good strategy to assume the whole input forces to the cardiac system are a Gaussian random variable, which represents the unobservable nature. The uncertainties of input forces could be removed or reduced if new observations of input forces can be reliably collected in clinical practice in the future. In the ideal case, where the input forces are fully known, the least-square filter will vanish, and the Kalman filter will be able to recover the motion from images directly.

2.3. Continuous State-Space Equations. In order to apply our simultaneous estimation strategy as the structure in [33], the dynamic equation (4) needs to be transformed into a continuous stochastic state equation first. Let the state vector be $x(t)=[U(t), \dot{U}(t)]^{T}$ and we can have

$$
\dot{x}(t)=A_{c} x(t)+B_{c} W(t)+n(t),
$$

where $n(t)$ is the process noise which is an additive, zeromean, white noise $\left(E[n(t)]=0 ; E\left[n(t) n(s)^{\prime}\right]=Q_{n}(t) \delta_{t s}\right.$, where $Q_{n}$ is the process noise covariance). The input forces $W(t)$, the system matrices $A_{c}$ and $B_{c}$ are

$$
\begin{aligned}
W(t) & =[R(t)], \\
A_{c} & =\left[\begin{array}{cc}
0 & I \\
-M^{-1} K & -M^{-1} C
\end{array}\right], \\
B_{c} & =\left[\begin{array}{c}
0 \\
-M^{-1}
\end{array}\right],
\end{aligned}
$$

the matrices $B_{c}$ and $W$ are not the same as the works in $[21,24]$ because we modify them for the estimation of input forces.

An associated measurement equation, which describes the observations provided by the images or imaging-derived data $y(t)$ can be expressed in the form:

$$
y(t)=H x(t)+e(t)
$$

where $e(t)$ is the measurement noise which is additive, zero mean, and white $\left(E[e(t)]=0 ; E\left[e(t) e(s)^{\prime}\right]=R_{e}(t) \delta_{t s}\right.$, where $R_{e}$ is the measurement noise covariance), independent of $n(t)$. Also, $H$ is the measurement matrix which should be specified by the relation between state vector $x(t)$ and measurement vector $y(t)$.

The process noise in (5) and the measurement noise in (7) are crucial in our stochastic approach. For example, linear elasticity is used in this work to approximate the dynamics of the myocardium. However, it is not the most realistic material model for myocardial dynamics. The distance between linear elasticity and real myocardial dynamics will contribute to the process noise in (5), as uncertainties in model. Other errors, such as discretization and initialization, also can be treated as uncertainties in computational model, that is, the process noise in (5). How to obtain the proper process noise is still a great challenge and active topic in many state-space approaches [40]. So is the measurement noise. The process noise and the measurement noise are adjusted manually in this work because the aim of this work is to establish a proper stochastic framework to address the issue of input forces. However, it is worthy to explore the properties and effects of the process noise and the measurement noise of this framework in future work.

2.4. Discrete State-Space Equations. The MR images are usually collected distinctly over the whole cardiac cycle, so (7) should be discretized according to corresponding imaging instants. However, (5) also needs to be discretized so that it can be run in a computer. It should be noted that the continuous-discrete Kalman filter has been proposed to recover continuous myocardial kinematic function in [41]. However, the state equation is still discretized in a very small time step to approximate the effect of continuous dynamics in [41]. We discretize (5) and (7) over the imaging sampling interval $T$. Since the imaging sampling interval $T$ is always a known constant, we can replace $k T$ with $k$ in

$$
\begin{gathered}
x(k+1)=A x(k)+B W(k)+n(k), \\
y(k)=H x(k)+e(k), \\
A=e^{A_{c} T}, \quad B=A_{c}^{-1}\left(e^{A_{c} T}-I\right) B_{c},
\end{gathered}
$$

$A$ and $B$ can be computed using Pade approximation [42]. The mathematical derivation of discrete state-space equations from continuous state-space equations is provided in [21]. Here if there are $N$ sample nodes to represent the myocardium, $A$ is a $4 N \times 4 N$ matrix, $B$ is a $4 N \times 2 N$ matrix, $x$ is a $4 N \times 1$ vector and $W$ is a $2 N \times 1$ vector.

2.5. Discrete State-Space Equations with Noisy Input Forces. In order to model the input forces as a random variable, typically seen in estimation and tracking literature [33, 43], Equation (8) are transformed into stochastic equations with noisy input forces:

$$
\begin{aligned}
x(k+1) & =A x(k)+B[W(k)+n(k)], \\
y(k) & =H x(k)+e(k),
\end{aligned}
$$

where $n(k)$ and $e(k)$ are the additive, zero-mean, white noises, but independent from each other. As can be seen in (10), the uncertainties in input forces $W(k)$ are modeled by putting $n(k)$ and $W(k)$ together. So the input forces are disturbed by the noise $n(k)$, which represents the unobservable nature of the input force. Though the dynamic of state $x$ is driven by the unobservable input forces now, we will provide an additional least-square filter to estimate the input forces and correct the estimation of state $x$. 


\section{Simultaneous Estimation of Myocardial Motion and Input Forces}

To handle unknown input forces in (10), we propose a framework of simultaneous estimation of myocardial kinematic function and input forces, which consists of two parts: a Kalman filter and a recursive least-square filter. Let $x^{-}, \hat{x}(k)$, and $\bar{x}(k)$ denote the prediction of the true state $x(k)$ without the input forces $W(k)$, the estimation of the true state $x(k)$ without the input forces $W(k)$, and the estimation of the true state $x(k)$ with the input forces $W(k)$, respectively. Then our proposed framework can be summarized below:

(1) Prediction without input forces:

$$
\begin{aligned}
& x^{-}(k)=A \bar{x}(k-1), \\
& P^{-}(k)=A \bar{P}(k-1) A^{T}+B Q_{n} B^{T} .
\end{aligned}
$$

(2) Update with measurements:

$$
\begin{aligned}
& S(k)=H P^{-}(k) H^{T}+R_{e}, \\
& G(k)=P^{-}(k) H^{T} S^{-1}(k), \\
& \bar{P}(k)=[1-G(k) H] P^{-}(k), \\
& \bar{z}(k)=Y(k)-H X^{-}(k), \\
& \bar{x}(k)=x^{-}(k)+G(k) \bar{z}(k),
\end{aligned}
$$

where the covariance of residual innovation sequence $\bar{z}(k)$ is $S(k)$.

(3) Estimation of input forces:

$$
\begin{aligned}
\Phi_{S}(k) & =H\left[A M_{S}(k-1)+I\right] B, \\
\Sigma & =\Phi_{S}(k) \gamma^{-1} P_{b}(k-1) \Phi_{S}^{T}(k)+S(k), \\
K_{b}(k) & =\gamma^{-1} P_{b}(k-1) \Phi_{S}^{T}(k) \Sigma^{-1}, \\
P_{b}(k) & =\left[I-K_{b} \Phi_{S}(k)\right] \gamma^{-1} P_{b}(k-1), \\
W(k) & =W(k-1)+K_{b}(k)\left[\bar{Z}(k)-\Phi_{S}(k) W(k-1)\right] .
\end{aligned}
$$

(4) Correction with input forces:

$$
\begin{aligned}
M_{s}(k) & =[I-G(k) H]\left[A M_{S}(k-1)+I\right], \\
\hat{x}(k) & =\bar{x}(k)+M_{S}(k) B W(k) .
\end{aligned}
$$

The detailed derivation of steps (3) and (4) can be found in the appendix of [33] However $\bar{P}(k)$ is the error covariance matrix of the Kalman filter without information of input forces, $S(k)$ is the residual innovation covariance, $G(k)$ is the Kalman gain, $\Phi_{S}(k)$ and $M_{S}(k)$ are the sensitivity matrices, $\bar{z}(k)$ is the residual innovation, $P_{b}(k)$ is the error covariance of the estimated input vector $W(k)$, and $K_{b}(k)$ is the correction gain for the updating $W(k)$. Also, $\Phi_{s}(k)$, $M_{s}(k), K_{b}(k)$, and $P_{b}(k)$ are a $4 N \times 2 N$ matrix, a $4 N \times 4 N$ matrix, a $4 N \times 4 N$ matrix, a $2 N \times 4 N$ matrix, and a $2 N \times 2 N$ matrix, respectively, if there are $N$ nodes in the triangular mesh of the myocardium. When $\gamma=1$, we will get the usual sequential least-square estimator, which is only suitable for a constant input force system. In the system with timevarying input forces, however, we like to prevent $K_{b}(k)$ from reducing to zero. This is accomplished by introducing the factor $\gamma$. By setting $0<\gamma<1, K_{b}(k)$ is effectively prevented from shrinking to zero. Hence, the corresponding leastsquare filter can preserve its updating ability continuously. However, the inherent data truncation effect brought by $\gamma$ causes variance increases in $W(k)$ in the estimation problem resulting from noise. Thus, it is necessary to compromise between fast adaptive capability and the loss of estimate accuracy.

Here, the Kalman filter is used to generate $G(k), S(k)$, and $\bar{z}(k)$, based on the state transition matrix $A$, input matrix $B$, and process and measurement noise covariance matrices $Q_{n}$ and $R_{e}$. The least-square filter is derived to compute the onset time histories of the unknown input forces by utilizing the Kalman gain, residual innovation covariance $S(k)$, and residual innovation $\bar{z}(k)$. In addition, our framework is initialized by setting $\bar{x}(-1)=0, W(-1)=0$, and $M_{S}(-1)=$ 0 . Since $\bar{P}(-1)=p \times I_{4 N \times 4 N}$ and $P_{b}(-1)=p_{b} \times I_{4 N \times 4 N}$ are normally assumed, where $I$ is the identity matrix, $p$ and $p_{b}$ are the constant scalar, we initialize $p$ and $p_{b}$ as large numbers, such as $10^{6}$ and $10^{2}$, respectively. This has the effect of treating the errors in the initial estimation of the input forces as large. However, after a few time steps, the estimation results should converge to their actual values rapidly if the state-space equations can capture the system dynamics quickly. This also shows that the present technique is not sensitive to the errors in the initial estimation. Though $Q_{n}$ and $R_{e}$ should be determined according to the noise level in the input forces and measurements, it is adjusted manually in this work according to empirical experiences.

\section{Experiments}

4.1. Synthetic Data. Our filtering strategy is first validated in synthetic data of a 2D object undergoing body forces only in the vertical direction ( $y$-axis) with the bottom being fixed. The magnitude of body forces in each triangular element is decided by the area of that element by setting the density of body forces to 1 Newton. Material properties and other parameters are taken as Young's modulus $E=75 \mathrm{kpa}$, Poisson's ratio $\nu=0.4$, damping coefficients $\alpha=0.01$ and $\beta=$ 0.1 . The simulation is generated by a general purpose finiteelement software, Abaqus. However 21 sampling frames of the motion are acquired, with displacements and velocities in all nodes, as the ground truth. Then Gaussian noises ( $20 \mathrm{~dB}$ ) are added in all nodes to generate noisy measurements. A single Kalman filter (KL) and our framework of the Kalman filter and the least-square filter (KLLS) are implemented for the estimation of kinematic function. In KL implementation, the nodal displacements with noise in the top of the synthetic object are used to construct input forces through the penalty method, which is the same as the work in [21]. The setting of process noise $Q_{n}$ and measurement noise $R_{e}$ is the same in KL and KLLS in order to maintain a fair comparison, where $Q_{n}=10^{-5}$ and $R_{e}=10^{-8}$. In KLLS, $\gamma$ is set to 0.82 . The displacement magnitude and strain maps of the ground truth 
TABLE 1: Differences between the ground truth and the KL/KLLS estimated nodal positions.

\begin{tabular}{lcccc}
\hline Method & Frame number & Maximum error & Mean error & Standard deviation \\
\hline \multirow{4}{*}{ KL } & number 4 & 0.181 & 0.063 & 0.036 \\
& number 8 & 0.146 & 0.074 & 0.037 \\
& number 12 & 0.168 & 0.086 & 0.043 \\
\hline \\
number 16 & 0.258 & 0.104 & 0.060 \\
KLLS & number 20 & 0.227 & 0.120 & 0.062 \\
& number 4 & 0.067 & 0.021 & 0.017 \\
& number 8 & 0.072 & 0.026 & 0.021 \\
& number 12 & 0.083 & 0.013 & 0.024 \\
& number 16 & 0.093 & 0.031 & 0.028 \\
\end{tabular}

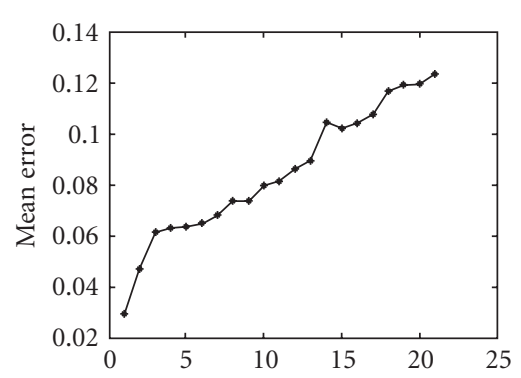

(a) mean error $(\mathrm{KL})$

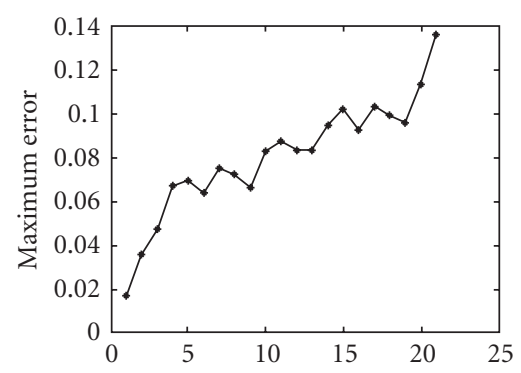

(d) max error (KLLS)

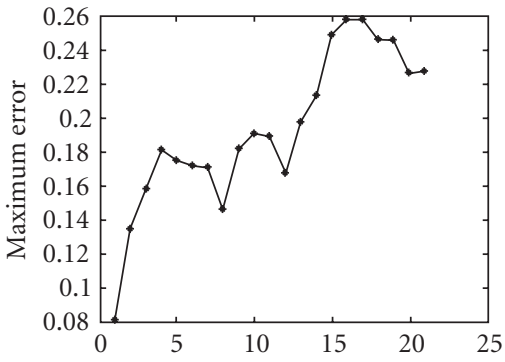

(b) max error (KL)

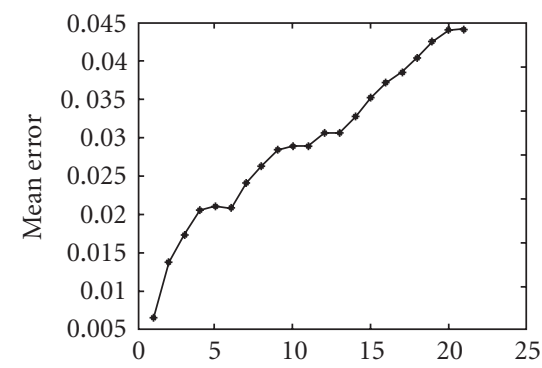

(e) mean error (KLLS)

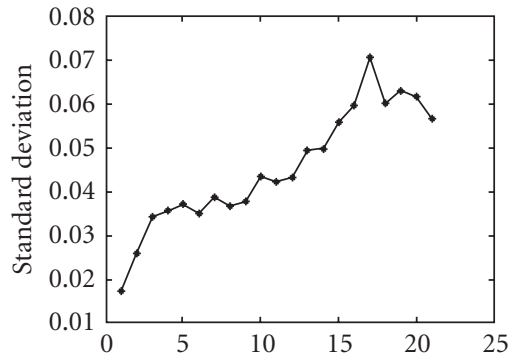

(c) $\mathrm{SD}$ error $(\mathrm{KL})$

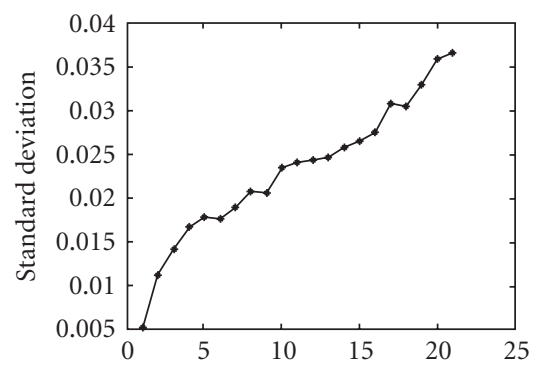

(f) $\mathrm{SD}$ error (KLLS)

Figure 2: (a) Mean, (b) max, and (c) standard deviation errors of the Kalman filter; (d) mean, (e) max, and (f) standard deviation errors of the Kalman filter and the least-square filter; all the $x$ axis in the figures are the numbering of frame.

and the estimated results for quantitative assessments and comparisons of two filtering strategies are shown in Figure 3. Overall point-by-point positional errors are measured by their mean and standard deviation. The mean is calculated by

$$
\text { Mean }=\frac{1}{N} \sum_{i}^{N}\left|\mathrm{Est}_{i}-\operatorname{Tru}_{i}\right| \quad i=1, \ldots, N
$$

where $N$ is the number of nodes, Est is the estimated nodal value and Tru is the true nodal value. The standard deviation is calculated by

$$
\mathrm{SD}=\sqrt{\frac{1}{N} \sum_{i}^{N}\left(\text { Est }_{i}-\text { Mean }^{2}\right.} \quad i=1, \ldots, N,
$$

where $N$ is the number of nodes and SD is the standard deviation. The growth of errors in KL and KLLS are illustrated in Figure 2, which shows errors of KLLS has stable behavior in comparison with KL. From Table 1, we also can see the quantitative measures of accumulated errors: such as in frame \#20, the maximum, mean and standard deviation of errors of KLLS are 0.114, 0.044, and 0.036; the maximum, mean and standard deviation of errors of $\mathrm{KL}$ are $0.227,0.120$, and 0.062 . The comparison between the estimated forces and ground truth are also shown in Table 2. Overall, our framework shows superior performance over the same measurements with the same noise level because of simultaneous estimation of kinematic function and input forces, but a single Kalman filter with boundary forces fails here because of wrong input forces dominated by noisy displacements in boundary. 

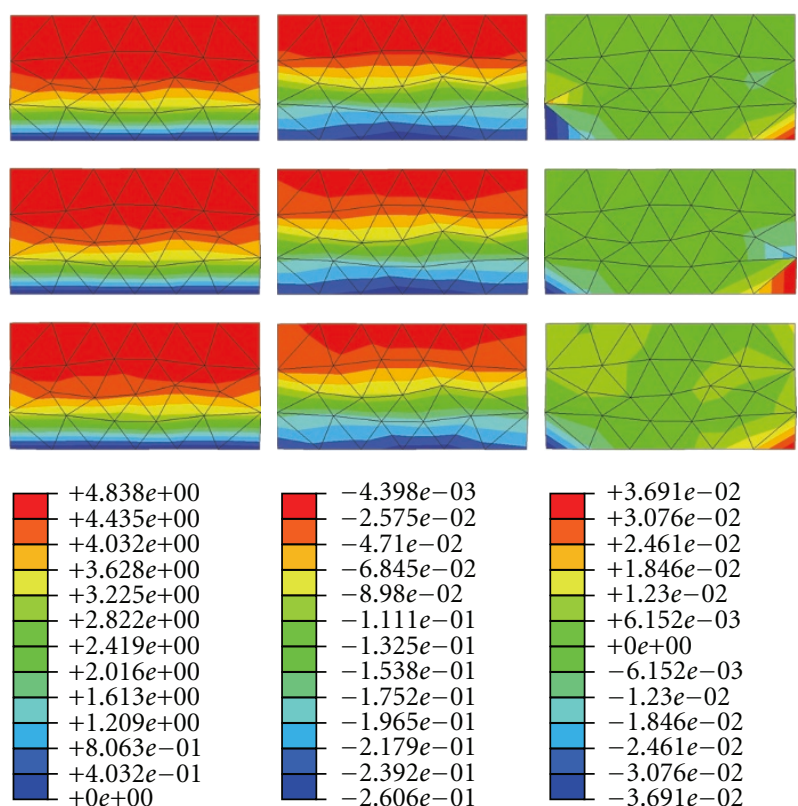

Figure 3: From first row to fourth row: ground truth, estimation results of our framework, estimation results of a single Kalman filter and color scale mapping. From first column to third column: magnitude maps of displacement, strain maps in $y$-axis and strain maps in $x y$-axis.

TABle 2: Comparison of the magnitude to total nodal forces between ground truth and estimation (KLLS).

\begin{tabular}{lcc}
\hline Frame number & Ground truth & Estimated result \\
\hline number 4 & $3.82 \mathrm{e}+003$ & $3.24 \mathrm{e}+003$ \\
number 8 & $3.81 \mathrm{e}+003$ & $3.22 \mathrm{e}+003$ \\
number 12 & $3.78 \mathrm{e}+003$ & $2.99 \mathrm{e}+003$ \\
number 16 & $3.73 \mathrm{e}+003$ & $2.99 \mathrm{e}+003$ \\
number 20 & $3.70 \mathrm{e}+003$ & $2.89 \mathrm{e}+003$ \\
\hline
\end{tabular}

4.2. Canine Image Data. The MR image data and representation of left ventricle (LV) are displayed in Figure 1. Myocardial displacements and velocities can be extracted using our previous multiframe algorithm [24] or other algorithms [19, 38, 44]. The infarcted tissue is highlighted in the triphenyl tetrazolium chloride (TTC) stained after mortem myocardium (Figure 1), which provides the clinical gold standard for the assessment of the image analysis results. The parameters of material properties of the myocardium are initialized as Young's modulus is set to $75 \mathrm{kpa}$, Poisson's ratio is set to 0.47 , damping coefficients $\alpha=0.01$ and $\beta=0.1$. The parameters of KLLS are initialized as the process noise $Q_{n}$ is set to $10^{-4}$, measurement noise is set to $10^{-10}$ and $\gamma$ is set to 0.53 . The estimated radial, circumferential, and RC shear strain maps are shown in Figure 4. The infarct tissue can be identified in the strain maps, and the most obvious difference is observed in the RC shear strain map, where the lower-right quarter of the myocardium has much larger strain than other normal tissues. These patterns are in good agreement with the highlighted TTC-stained tissue in Figure 1, demonstrating the clinical relevance of our strategy.

\section{Conclusion}

In this paper, we have presented a biomechanically constrained filtering framework for the multiframe estimation of the nonrigid cardiac kinematics from medical image sequence. In spite of linear elasticity used to approximate myocardial system dynamics, our framework could allow convenient incorporation of other material constraints. The input estimation filtering formulation facilitates the considerations of input data uncertainty, and the Kalman filtering principles are adopted to achieve optimal estimation of the myocardial kinematics over corrected input forces. Quantitative validation has been conducted using synthetic data with known ground truth, and physiological experiment results are acquired from MR image sequences, as validated by post-mortem tissue staining.

The conventional Kalman filtering strategy consists of two steps: prediction and correction. The successful place of the conventional Kalman filtering strategy is computing the estimated state variables between predictions and measurements in minimum mean square sense. However, the predictions generated by the model could be faulty if the input term of the model is wrongly added, and then the error of estimations could be dominated by the imperfect predictions. In the case of myocardial dynamics, it will demand arduous work, including tremendous experiments, to determine patient's loading condition, which is not feasible in daily clinical practice. So it is reasonable to treat the loading, that is, input forces, of the heart as stochastic sources. In our particular application, it can be easily seen that the response of the cardiac system will vary largely with different loading conditions from (4). The forces constructed from boundaries or image features are obviously different from the real situation in the myocardium and a minor error from these image-derived forces could be amplified by (4) easily. Since the patient-specific observations of input forces in the cardiac system are still unavailable, it is meaningful to handle the uncertainties in input forces and measurements simultaneously, which can increase the accuracies of estimated state variables if estimated input forces are closed to ground truth.

Though this approach is inspired by [33] from the heating problem, it is closely related with previous state space approaches [21, 30, 41]. Works like [21, 30, 41] could be considered as one special situation of our approach where least-square filter vanishes if the external loading can be specified deterministically. The demanding computation also prohibits the performance of our filtering strategy in 3D because of burdensome computation of the Kalman filter and extra calculation of the least-square filter. Since the computation of the conventional 3D Kalman filter could be properly reduced by applying model reduction [25] or reduced rank filter [45], it will be worthy to apply similar techniques in our work. Further, the material properties of the biomechanical models may be estimated along with the motion properties through the augmentation of the state 

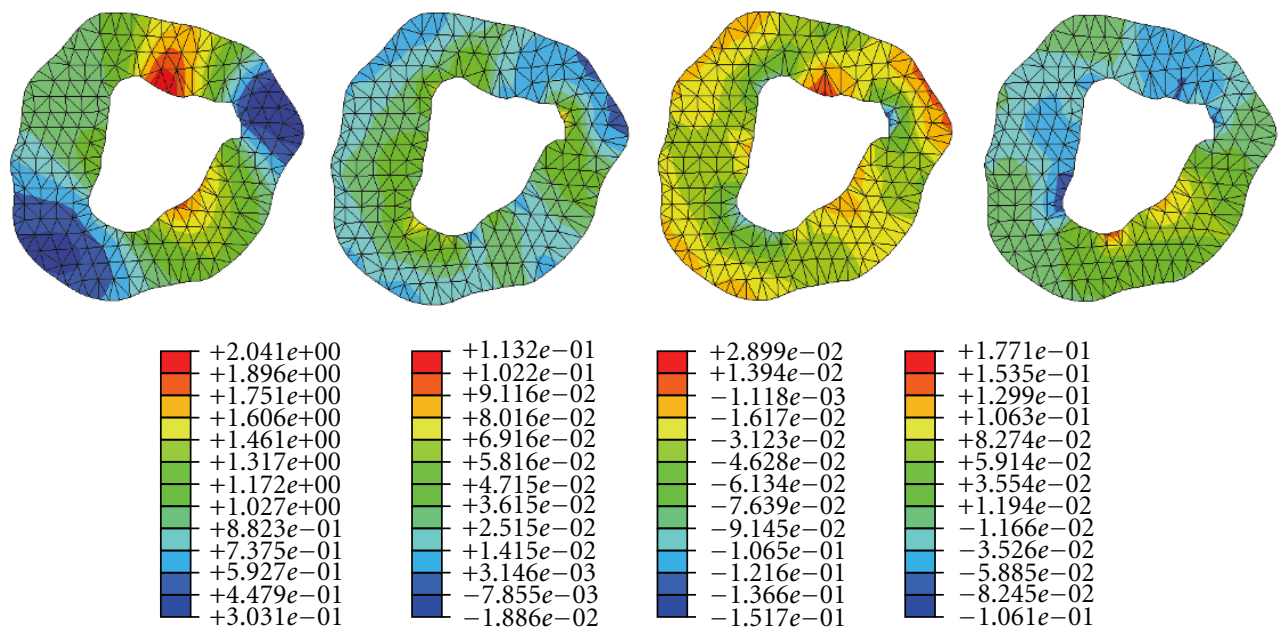

FIGURE 4: From left to right: estimated displacement magnitude, radial, circumferential, and RC shear strain maps for frame \#9 (with respect to frame number 1 ).

vector by the material parameters and the construction of the nonlinear augmented state-space representation [24].

\section{Acknowledgments}

This work is supported by The Wellcome Trust Fund. The authors would like to extend their gratitude to Dr. Albert Sinusas of Yale University for the canine experiment and imaging data. The valuable comments and suggestions from the anonymous reviewers are also greatly appreciated.

\section{References}

[1] S. C. Amartu and H. J. Vesselle, "A new approach to study cardiac motion: the optical flow of cine MR images," Magnetic Resonance in Medicine, vol. 29, no. 1, pp. 59-67, 1993.

[2] M. J. Ledesma-Carbayo, J. Kybic, M. Desco, et al., "Spatiotemporal nonrigid registration for ultrasound cardiac motion estimation," IEEE Transactions on Medical Imaging, vol. 24, no. 9, pp. 1113-1126, 2005.

[3] S. Song and R. Leahy, "Computation of 3D velocity fields from. 3D cine CT images," IEEE Transactions on Medical Imaging, vol. 10, no. 3, pp. 295-306, 1991.

[4] A. A. Amini, Y. Chen, M. Elayyadi, and P. Radeva, "Tag surface reconstruction and tracking of myocardial beads from SPAMM-MRI with parametric b-spline surfaces," IEEE Transactions on Medical Imaging, vol. 20, no. 2, pp. 94-103, 2001.

[5] T. S. Denney, "Estimation and detection of myocardial tags in MR image without user-defined myocardial contours," IEEE Transactions on Medical Imaging, vol. 18, no. 4, pp. 330-344, 1999.

[6] M. A. Guttman, J. L. Prince, and E. R. McVeigh, "Tag and contour detection in tagged MR images of the left ventricle," IEEE Transactions on Medical Imaging, vol. 13, no. 1, pp. 7488, 1994.

[7] A. A. Amini and J. S. Duncan, "Bending and stretching models for LV wall motion analysis from curves and surfaces," Image and Vision Computing, vol. 10, no. 6, pp. 418-430, 1992.
[8] P. C. Shi, A. J. Sinusas, R. T. Constable, E. Ritman, and J. S. Duncan, "Point-tracked quantitative analysis of left ventricular surface motion from 3-D image sequences," IEEE Transactions on Medical Imaging, vol. 19, no. 1, pp. 36-50, 2000.

[9] J. C. McEachen II and J. S. Duncan, "Shape-based tracking of left ventricular wall motion," IEEE Transactions on Medical Imaging, vol. 16, no. 3, pp. 270-283, 1997.

[10] N. J. Pelc, M. Drangova, L. R. Pelc, et al., "Tracking of cyclical motion using phase contrast cine MRI velocity data," Journal of Magnetic Resonance Imaging, vol. 5, pp. 339-345, 1995.

[11] J. Huang, D. Abendschein, V. G. Davila-Roman, and A. A. Amini, "Spatio-temporal tracking of myocardial deformations with a 4-D B-spline model from tagged MRI," IEEE Transactions on Medical Imaging, vol. 18, no. 10, pp. 957-972, 1999.

[12] T. S. Denney and J. L. Prince, "Reconstruction of 3D left ventricular motion from planar tagged cardiac MR images: an estimation theoretic approach," IEEE Transactions on Medical Imaging, vol. 14, no. 4, pp. 625-635, 1995.

[13] S. Benayoun and N. Ayache, "Dense non-rigid motion estimation in sequences of medical images using differential constraints," International Journal of Computer Vision, vol. 26, no. 1, pp. 25-40, 1998.

[14] A. P. Pentland and B. Horowitz, "Recovery of nonrigid motion and structure," IEEE Transactions on Pattern Analysis and Machine Intelligence, vol. 13, no. 7, pp. 730-742, 1991.

[15] S. Sclaroff and A. P. Pentland, "Modal matching for correspondence and recognition," IEEE Transactions on Pattern Analysis and Machine Intelligence, vol. 17, no. 6, pp. 545-561, 1995.

[16] R. T. Constable, P. C. Shi, A. Sinusas, and J. Duncan, "Volumetric deformation analysis using mechanicsbased data fusion: applications in cardiac motion recovery," International Journal of Computer Vision, vol. 35, no. 1, pp. 87-107, 1999.

[17] D. P. Dione, R. T. Constable, X. Papademetris, A. J. Sinusas, and J. S. Duncan, "Estimation of 3D left ventricular deformation from echocardiography," Medical Image Analysis, vol. 5, pp. 17-28, 2001.

[18] D. P. Dione, R. T. Constable, X. Papademetris, A. J. Sinusas, and J. S. Duncan, "Estimation of 3D left ventricular deformation from medical images using biomechanical models," IEEE 
Transactions on Medical Imaging, vol. 21, no. 7, pp. 786-799, 2002.

[19] J. S. Duncan and N. Ayache, "Medical image analysis: progress over two decades and the challenges ahead," IEEE Transactions on Pattern Analysis and Machine Intelligence, vol. 22, no. 1, pp. 85-106, 2000.

[20] A. F. Frangi, W. J. Niessen, and M. A. Viergever, "Threedimensional modeling for functional analysis of cardiac images," IEEE Transactions on Medical Imaging, vol. 20, no. 1, pp. 2-25, 2001.

[21] H. F. Liu and P. C. Shi, "State-space analysis of cardiac motion with biomechanical constraints," IEEE Transactions on Image Processing, vol. 16, no. 4, pp. 901-917, 2007.

[22] Y. Bar-Sharlom, X. Li, and T. Kirubarajan, Estimation with Applications to Tracking and Navigation, John Wiley \& Sons, New York, NY, USA, 2000.

[23] H. F. Liu and P. C. Shi, "Cardiac motion and elasticity characterization with iterative sequential hinfinity criteria," in Medical Image Computing and Computer Assisted Intervention, pp. 34-42, 2004.

[24] P. C. Shi and H. F. Liu, "Stochastic finite element framework forsimultaneous estimation of cardiac kinematic functions and material parameters," Medical Image Analysis, vol. 7, no. 4, pp. 445-464, 2003.

[25] K. C. L. Wong, L. Wang, H. Zhang, H. F. Liu, and P. C. Shi, "Computational complexity reduction for volumetric cardiac deformation recovery," Journal of Signal Processing Systems, vol. 55, no. 1-3, pp. 281-296, 2009.

[26] P. Moireau, D. Chapelle, and P. Le Tallec, "Filtering for distributed mechanical systems using position measurements: perspectives in medical imaging," Inverse Problems, vol. 25, no. 3, Article ID 035010, 25 pages, 2009.

[27] P. Moireau, D. Chapelle, and P. Le Tallec, "Joint state and parameter estimation for distributed mechanical systems," Computer Methods in Applied Mechanics and Engineering, vol. 197, no. 6-8, pp. 659-677, 2008.

[28] D. Chapelle and P. Moireau, "Robus filter for joint stateparameters estimation in distributed mechanical system," Discrete and Continous Dynamical Systems, vol. 23, no. 1-2, pp. 65-84, 2009.

[29] L. Ljung, "Asymptotic behavior of the extended kalman filter as a parameter estimator for linear systems," Computer Methods in Applied Mechanics and Engineering, vol. 24, no. 1, pp. 36-50, 1979.

[30] C. L. Wong, H. Y. Zhang, and P. C. Shi, "Physiome model based state-space framework for cardiac kinematics recovery," in Medical Image Computing and Computer Assisted Intervention, pp. 720-727, 2006.

[31] M. Sermesant, H. Delingette, and N. Ayache, "An electromechanical model of the heart for image analysis and simulation," IEEE Transactions on Medical Imaging, vol. 25, no. 5, pp. 612$625,2006$.

[32] Z. H. Hu, D. Metaxas, and L. Axel, "In-vivo strain and stress estimation of the left ventricle from MRI images," in Medical Image Computing and Computer Assisted Intervention, pp. 706$713,2002$.

[33] P. C. Tuan, C. C. Ji, L. W. Fong, and W. T. Huang, "An input estimation approach to on-line two-dimensional inverse heat conduction problems," Numerical Heat Transfer, Part B, vol. 29, no. 3, pp. 345-363, 1996.

[34] L. Glass, P. Hunter, and A. McCulloch, Theory of Heart: Biomechanics, Biophysics, and Nonlinear Dynamics of Cardiac Function, Springer, New York, NY, USA, 1991.
[35] K. J. Bathe, Finite Element Procedures, Prentice-Hall, Englewood Cliffs, NJ, USA, 1996.

[36] H. Yamada, Strength of Biological Material, Williams and Wilkins, Baltimore, Md, USA, 1970.

[37] L. N. Wong, H. F. Liu, A. J. Sinusas, and P. C. Shi, "Simultaneous recovery of left ventricular shape and motion using meshfree particle framework," in Proceedings of IEEE International Symposium on Biomedical Imaging: From Nano to Macro, pp. 1263-1266, 2004.

[38] B. Li, A. A. Young, and B. R. Cowan, "Validation of GPU accelerated non-rigid registration for the evaluation of cardiac function," in Medical Image Computing and Computer Assisted Intervention, pp. 880-887, 2008.

[39] L. W. Wang, H. Y. Zhang, K. C. L. Wong, H. F. Liu, and P. C. Shi, "Noninvasive imaging of 3D cardiac electrophysiology," in Proceedings of IEEE International Symposium on Biomedical Imaging: From Nano to Macro, pp. 632-635, 2006.

[40] P. F. J. Lermusiaux, "Adaptive modeling, adaptive data assimilation and adaptive sampling," Physica D, vol. 230, no. 1-2, pp. 172-196, 2007.

[41] C. L. Wong, H. Y. Zhang, and P. C. Shi, "Cardiac motion recovery: continuous dynamics, discrete measurements, and optimal estimation," in Medical Image Computing and Computer Assisted Intervention, pp. 744-751, 2006.

[42] G. H. Golub and C. F. Van Loan, Matrix Computation, Johns Hopkins University Press, Baltimore, Md, USA, 1983.

[43] T. Glad and L. Ljung, Control Theory, Taylor \& Francis, London, UK, 2000.

[44] E. Haber, D. N. Metaxas, and L. Axel, "Motion analysis of the right ventricle from MRI images," in Medical Image Computing and Computer Assisted Intervention, pp. 177-188, 1998.

[45] D. T. Pham, J. Verron, and M. C. Roubaud, "Singular evolutive extended kalman filter with eof initialization for data assimilation in oceanography," Journal of Marine Systems, vol. 16, no. 3-4, pp. 323-340, 1998. 